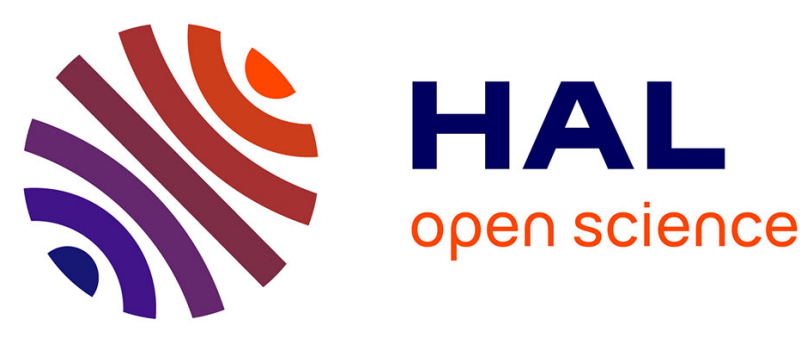

\title{
Time Is Not Money
}

Tore Ellingsen, Magnus Johannesson

\section{To cite this version:}

Tore Ellingsen, Magnus Johannesson. Time Is Not Money. Journal of Economic Behavior and Organization, 2009, 72 (1), pp.96. 10.1016/j.jebo.2009.05.010 . hal-00699366

\section{HAL Id: hal-00699366 https://hal.science/hal-00699366}

Submitted on 21 May 2012

HAL is a multi-disciplinary open access archive for the deposit and dissemination of scientific research documents, whether they are published or not. The documents may come from teaching and research institutions in France or abroad, or from public or private research centers.
L'archive ouverte pluridisciplinaire HAL, est destinée au dépôt et à la diffusion de documents scientifiques de niveau recherche, publiés ou non, émanant des établissements d'enseignement et de recherche français ou étrangers, des laboratoires publics ou privés. 


\section{Accepted Manuscript}

Title: Time Is Not Money

Authors: Tore Ellingsen, Magnus Johannesson

PII: $\quad$ S0167-2681(09)00147-4

DOI: $\quad$ doi:10.1016/j.jebo.2009.05.010

Reference: $\quad$ JEBO 2398

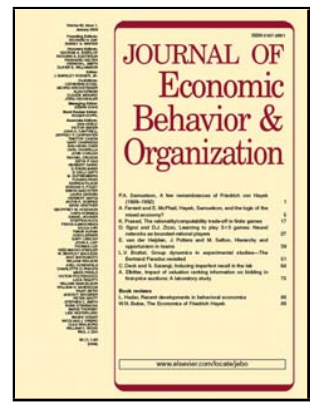

To appear in: Journal of Economic Behavior \& Organization

Received date: $\quad$ 7-12-2006

Revised date: $\quad 7-5-2009$

Accepted date: $\quad$ 8-5-2009

Please cite this article as: Ellingsen, T., Johannesson, M., Time Is Not Money, Journal of Economic Behavior and Organization (2008), doi:10.1016/j.jebo.2009.05.010

This is a PDF file of an unedited manuscript that has been accepted for publication. As a service to our customers we are providing this early version of the manuscript. The manuscript will undergo copyediting, typesetting, and review of the resulting proof before it is published in its final form. Please note that during the production process errors may be discovered which could affect the content, and all legal disclaimers that apply to the journal pertain. 


\title{
Time Is Not Money
}

\author{
Tore Ellingsen* \\ Stockholm School of Economics \\ Magnus Johannesson** \\ Stockholm School of Economics
}

May 7, 2009

\begin{abstract}
In an ultimatum bargaining experiment, we study how subjects bargain over the returns to their investments of money and time. The most notable finding is that a third of the subjects demand no compensation for their time investments, whereas almost all subjects demand compensation for equally costly monetary investments.
\end{abstract}

JEL Classification: C91, J20, L14, Z13.

KEYWORDS: Bargaining, sunk costs, time domain, monetary domain.

${ }^{*}$ Corresponding author. Address: Department of Economics, Stockholm School of Economics, Box 6501, S-113 83 Stockholm, Sweden. Email: gte@hhs.se. Phone: +46-8736 9260. Fax: +46-8-31 3207.

**Address: Department of Economics, Stockholm School of Economics, Box 6501, S11383 Stockholm, Sweden. Email: hemj@hhs.se.

We are grateful to Erik Grönqvist and Niklas Zethraeus for research assistance and to the Jan Wallander and Tom Hedelius Foundation for generous monetary donations. Thanks to Simon Gächter, Andrew Postlewaite, Lisa Román, Jean-Robert Tyran, two anonymous referees, and seminar participants at University of Gothenburg and University of St. Gallen for donating some of their valuable time to us. Errors are ours. 


\section{Introduction}

Human capital theory, as developed by Becker $(1964,1965)$ and others, has been tremendously successful at explaining many features of social interaction. However, by focussing on the exchange of labor services for money, and assuming convertibility between the two, human capital theory takes for granted Benjamin Franklin's dictum that "time is money." By contrast, anthropologists have long insisted that the convertibility between time and money is circumscribed by norms and values. Many societies place tight restrictions on exchange, several of the most striking examples being recounted by Bohannan (1963, Part 4). ${ }^{1}$ However, since many of the anthropologists' favorite examples concern "exotic" cultures, their impact on mainstream economic thinking has been modest. In this paper, we provide experimental evidence that Swedish students also reason somewhat differently about their time than about their money.

Our experiment runs as follows. One party, let us call her the proposer, can make an investment that yields a potential monetary gain from trade with another party, let us call him the responder. Some proposers can make a monetary investment, others invest time. Upon investment, the proposer makes a bargaining proposal concerning how to split the monetary gain. The responder, whose gain from trade is known, either accepts or rejects the proposal. The primary question is whether the proposal is different under time investment than under monetary investment.

For the comparison to be valid, costs and benefits must be as similar as possible across the two treatments. Since the opportunity cost of a proposers' time is not observed by the responder, the opportunity cost of monetary investments should also not be observed by responders - preferably, the two investment cost distributions should be identical. To achieve comparability, we elicit a distribution of time costs from a separate group of subjects, with the same characteristics. In the monetary treatment, both proposers and responders know that the investment costs are drawn from the elicited distribution, but only proposers learn the realization. Using the elicited distribution of investment costs, it is straightforward to keep the two treatments comparable. In the non-monetary treatment, the investment is to perform a task for 20 minutes. In the monetary treatment, the monetary investment

\footnotetext{
${ }^{1}$ The notion that peoples' behavior is "domain-specific" is quite common in other social sciences. See for example Waltzer (1983) and Tetlock et al. (2000). For a sociological discussion of the meaning of money along similar lines, see Zelitzer (1994).
} 
cost is drawn from an elicited distribution of time costs. The proposer's investment always generates a potential gain from trade of SEK 100.

If agents are selfish, theory predicts that proposers should always invest and make a proposal of SEK 99 (as long as the investment cost is below SEK 100). However, a large body of work in experimental economics suggests that a substantial fraction of individuals have strong preferences for fairness in bargaining situations, and that proposers often propose an equal split (see Camerer (2003) and Fehr and Schmidt (1999) for overviews). It has also been shown that, with monetary investment costs, subjects often propose an equal split of the net surplus. That is, they split evenly the surplus remaining after compensating proposers for the sunk investment cost (Ellingsen and Johannesson 2004a, 2005a). ${ }^{2}$ With a non-zero investment cost even proposers that care strongly about fairness are therefore predicted to make proposal above SEK 50.

All the models referred to above as well as the various recent models of social preferences and reciprocity (Rabin, 1993; Fehr and Schmidt, 1999; Bolton and Ockenfels, 2000; Charness and Rabin, 2002) predict that behavior should be independent of whether the proposer invests time or money.

Contrary to this prediction, we find that behavior differs markedly between the two experimental treatments. In the monetary treatment almost all proposers claim more than half the gross gain from trade. In the nonmonetary treatment, about a third of the proposers divide the gross gain from trade equally, despite having incurred a positive investment cost.

\section{Experiment}

Our subjects were recently enrolled undergraduate business and economics students at the Stockholm School of Economics. They were paid a participation fee of SEK 100, and they could earn between SEK 0 and SEK 100 in the experiment (SEK is Swedish Kronor; SEK 10 was approximately USD 1 at the time of the experiment). A total of 276 subjects participated in the experiment (i.e. 138 bargaining pairs). Five sessions were carried out with between 26 and 29 bargaining pairs in each session (one session with

\footnotetext{
${ }^{2}$ Ellingsen and Johannesson 2005 b conducted a similar study with a non-monetary investment cost. However, the monetary equivalent of the investment cost was elicited without giving incentives for truthful reporting, and the paper did not consider a comparison between monetary and non-monetary investments.
} 
Treatment I and two sessions each with Treatment II and Treatment III). Each subject participated in one treatment only. The three experimental treatments, referred to as the "control group", the "time investment group", and the "money investment group" in the text, are described in more detail below. The full instructions are available as an online appendix.

An important problem in experimental work is that the framing of the experiment matters. If the frame strongly suggests that the experiment is concerned with gift giving, that by itself can trigger gift giving behavior. Since we wanted to focus on generous behavior in situations where generosity is not obviously called for, we framed the experiment as a bargaining problem.

In the experiment two individuals, form a pair. In our instructions, the individuals are referred to neutrally as individuals $\mathrm{A}$ and $\mathrm{B}$, but for convenience we shall here call them respectively proposers and responders. Proposers and responders are located in different rooms, room A and room B, and pairing is anonymous and random. The proposer first decides whether to carry out an investment or not. If the investment is carried out a SEK 100 potential gain from trade is created for the pair, and the proposer and the responder bargain over how to divide the SEK 100 between them in an ultimatum game. The proposer suggests a division and the responder accepts or rejects this division. If the proposal is accepted the SEK 100 is divided according to the proposal, and if the proposal is rejected both get nothing. To compare monetary and non-monetary investment costs, we implement three experimental treatments that vary with respect to the investment cost. In the first ("control") treatment the investment cost is zero, which imply that subjects essentially play the standard ultimatum game. In the second ("time") treatment the investment is for the proposer to stay for 20 minutes after the end of the experiment. The investment cost in the third ("monetary") treatment consists of a monetary cost that is equivalent to the cost of staying 20 minutes after the end of the experiment. Because the time cost of 20 minutes will vary between proposers, and the cost is private information to the proposer, we separately elicit a distribution for the time cost and randomly draw a monetary cost from this distribution for each proposer in the third treatment. The monetary investment cost is private information to the proposer, and the responder only knows the procedure for determining it.

The distribution of time costs is elicited from the subjects in the control treatment after they have completed the ultimatum game, using the BeckerDeGroot-Marschak (BDM) mechanism (Becker, DeGroot, and Marschak, 1964). That is, each subject is asked to state a reservation price for staying 
20 minutes. Then a random number $x$ is drawn. If the subject's reservation price is below $x$, the subject is paid $x$ and must stay. Otherwise, the subject leaves.

We now describe the three treatments in more detail.

\subsection{Treatment I: Costless investment (control)}

In each of the two rooms subjects are given a number between 1 and $N$, where $\mathrm{N}$ is the number of students in the room. Subjects with identical numbers form a pair. Matching is random, and anonymity is maintained throughout. The subjects are given the instructions and are asked to read them. Subjects are allowed to ask clarifying questions to the experimenter, but not publicly. At the beginning of the instructions the subjects are told whether they are in room $\mathrm{A}$ or room $\mathrm{B}$.

After the subjects have read the instructions the subject in room $\mathrm{A}$ (the proposer) decides whether he/she will carry out an investment or not, at a zero investment cost. If the investment is carried out, a SEK 100 revenue is created for the pair, and the proposer proposes how to split it. If the subject in room B (the responder) accepts the proposal, both individuals are paid according to the proposal. If the responder rejects the proposal, both get nothing, except their participation fees.

\subsection{Treatment II: Time investment}

Treatment II is identical to Treatment I with the exception that the investment for the proposer now consists of staying for 20 minutes after the end of the experiment. After the subjects have been paid, all responders leave. The proposers that decided not to invest also leave at this point. After 20 minutes the investors leave.

\subsection{Treatment III: Money investment}

Treatment III is identical to Treatment II with the exception that the investment is now monetary. All subjects are told that the investment cost varies between the proposers and that the size of the investment cost is communicated only to the respective proposer. All subjects are also given the following information about how the investment cost has been determined: "In a separate group in the experiment 50 individuals have valued their cost 
of staying after the end of the experiment for 20 minutes. The valuation was done in a way so that every person had an incentive to reveal his/her true cost. For every person in room A we have randomly chosen the cost of giving up 20 minutes for one of these 50 individuals and used this cost as the investment cost."

Two remarks are in order. First, we know from Ellingsen and Johannesson (2005a) that responders' expectations about proposers' sunk costs matter for responders' behavior. If we would not have explained how the investment cost distribution is generated, there is thus little reason to assume that responders in the money treatment would behave as in the time treatment. Second, note that a proposer still has to undertake the investment in case the bargaining proposal is rejected. Thus, it is possible for a proposer to leave the experiment with less than the SEK 100 participation fee. We correctly anticipated that there would be no instances in which the time cost exceeded SEK 100 and the proposer invested. Hence, we never had to ask subjects to pay out of their own pocket.

\section{$2.4 \quad$ Eliciting time costs}

The subjects that participated in Treatment I also valued the cost of staying for 20 minutes. Before the experiment started they were told that they would participate in two experiments. When they had completed the first experiment, Treatment I, they were given the instructions for the second experiment, valuing the cost of 20 minutes. We used this procedure because we wanted the valuation of the time cost to resemble as closely as possible the situation in the time investment group (Treatment II).

The BDM mechanism, developed by Becker, DeGroot, and Marschak (1964), provides incentives for subjects to truthfully reveal the value they assign to a private good, in this case 20 minutes of the subjects' time. Each subject states his/her minimum compensation (price) for staying 20 minutes after the end of the experiment (and they are told that they will complete a questionnaire during these 20 minutes to pass the time). We draw a buying price randomly from a uniform distribution between SEK 0 and SEK 100 (with SEK 5 increments). If the buying price exceeds or equals the reservation price, the subject receives the buying price and stays for 20 minutes. If the buying price is below the reservation price the subject leaves. As in previous applications of the BDM mechanism (e.g. Grether and Plott 1979; Bohm, Lindén, and Sonnegård 1997) the subjects were informed about the 
incentive compatibility properties of the BDM mechanism. As recommended by Bohm, Lindén, and Sonnegård (1997), we did not communicate the upper bound of the buying price. Instead, we told the subjects that the upper bound is set at a level corresponding to the maximum reasonable valuation of the cost of giving up 20 minutes. $^{3}$

\subsection{Hypotheses and tests}

Our null hypothesis is that bargaining behavior is the same in the three treatments. Because bargaining experiments usually lead to skewed distributions, we employ bootstrapped standard errors when comparing mean proposals across treatments. ${ }^{4}$ The significance levels for the comparisons of proposals have all been obtained by generating 2,099 bootstrap replications. To compare proportions (e.g., the proportion of investors and the proportion of accepted proposals) between treatments, we use a contingency table Pearson chi-square test (D'Agostino, Chase, and Belanger 1988). All reported p-values are two-sided. Finally, we use logistic regression analysis to test whether the responders' acceptance probability differs across treatments, controlling for the bargaining proposal.

\section{Results}

Figure 1 displays the distribution of elicited time costs. The time cost varies between SEK 0 and SEK 100 for the 56 subjects. ${ }^{5}$ The mean value is SEK 28 and the median value is SEK 25, which is also the most common time cost - about $20 \%$ of the subjects had a time cost of SEK 25. The distribution of time costs in Treatment III is virtually identical to that in Figure $1 .^{6}$

\footnotetext{
${ }^{3}$ Incidentally, our upper bound turned out to coincide exactly with the highest reported valuation.

${ }^{4}$ See e.g. Efron and Tibshirani (1993) and Davidson and MacKinnon (1999). As we shall see, our sample is large enough to bring the resulting $p$-values fairly close to the $p$-values associated with the $t$-test, whereas the distance to non-parametric test statistics is large (due to skewness); see footnote ??.

${ }^{5}$ All time costs except two were given in even SEK 5 increments. The two time costs that were not in even SEK 5 increments (SEK 39 and SEK 49) are included in the closest categories in Figure 1.

${ }^{6}$ All the estimated time costs in Figure 1 is used except one estimate, because the number of estimates $(n=56)$ is one more than the bargaining pairs $(n=55)$. The (randomly
} 
Observe that the compensation i the experiment is tax free. For comparison, the average hourly earnings of wage earners in wholesale and retail trade (which is the kind of job that many students will hold part-time) was roughly SEK 104 before tax. $^{7}$ The twenty minute equivalent is SEK 34.7 before tax, corresponding to about SEK 24.3 after deducting the relevant marginal tax of thirty percent. The elicited values are thus well in line with the wage measure of the opportunity cost of time.

$$
\text { ****INSERT FIGURE } 1 \text { ABOUT HERE**** }
$$

\subsection{Proposer behavior}

Table 1 reports the "average" behavior of proposers and responders as well as the share of proposals weakly below 50 and weakly above $80 .{ }^{8}$ Proposers in the money investment group on average make proposals that are about SEK 5 higher than the proposals in the time investment group $(\mathrm{p}=0.056)$, and SEK 9 higher than the proposals in the control group $(\mathrm{p}=0.001)$. Although the average proposal is somewhat higher in the time investment group than in the control group, we cannot reject the null hypothesis of no difference $(p=0.213)$.

******** INSERT TABLE 1 ABOUT HERE**********

There is also a tendency that fewer proposers are willing to make monetary investments. In the time investment group only one proposer abstained from making the investment, whereas four proposers abstained from making the investment in the money investment group. Again the difference is not statistically significant $(p=0.170)$. As might be expected, the four proposers that abstain from investing have relatively high investment costs (SEK 100, SEK 70, SEK 50 and SEK 25). This is consistent with Ellingsen and Johannesson (2005a), who showed that asymmetric information about investment costs reduces investment compared to various complete information benchmarks.

\footnotetext{
chosen) estimate that is not used is SEK 80.

${ }^{7}$ Table 340, Statistical Yearbook of Sweden 2004, the columns for year 2002 (when the experiment was conducted).

${ }^{8}$ As explained above, we use bootstrapped standard errors when making comparisons across treatments. If we instead use a Mann-Whitney test to compare average proposals, the corresponding $p$-values are $0.102,<0.001$, and 0.212 (instead of $0.213,0.001$, and 0.056). The difference is that the Mann-Whitney test only uses ordinal information. An independent samples $t$-test yields $p$-values closer to the bootstrapped values, namely 0.236 , 0.003 , and 0.063 .
} 
There is a tendency for proposers with higher investment costs to demand a larger share of the SEK 100. An increase in the investment cost by SEK 1 in the money investment group on average increases the proposal by SEK $0.16(p=0.096) .^{9}$

Figure 2 displays the distribution of proposals in the control group (the ultimatum game). ${ }^{10}$ The most common proposals are SEK 50 and SEK 60, which is well in line with other studies; see Fehr and Schmidt (1999) and Camerer (2003, Chapter 2).

$* * * * * * * * * * * * *$ INSERT FIGURE 2 ABOUT HERE

Figure 3 compares the distribution of proposals between the money and time investment groups. ${ }^{11}$

**************INSERT FIGURE 3 ABOUT HERE*******************

Comparing behavior in the time treatment to that in the money treatment using a Two-Sample Kolmogorov-Smirnov test, we find that the two distributions are significantly different $(p=0.038)$. The most striking difference is the proportion of 50/50 proposals. The proportion of equal splits of the gross surplus is $30 \%$ in the time investment group, but only $6 \%$ in the money investment group. This difference is highly significant $(p=0.002)$. Interestingly, two subjects in the time investment group propose SEK 0, i.e. they give away all the gains from trade. At the same time, there appears to be a countervailing tendency towards high proposals in the time investment group. The fraction of proposals at SEK 80 or above is $28 \%$ in the time investment group and $18 \%$ in the money investment group, but the difference is not statistically significant $(p=0.217)$.

To the extent that subjects in the money investment group opt for an equal split, they do so after accounting for their investment cost. More precisely, $18 \%$ of the subjects split the net surplus exactly equally, $29 \%$ are within SEK 2.5 of an equal split (the relevant comparison when subjects round their offers to the nearest SEK 5), and $41 \%$ are within SEK 5 of

\footnotetext{
${ }^{9}$ In Ellingsen and Johannesson (2005a) $75 \%$ of proposers had zero investment cost and $25 \%$ had an investment cost of SEK 60. Despite this difference in the investment cost distribution, the relationship between the size of the investment cost and the bargaining proposal is quite similar across the two studies. There, an increase in the investment cost by SEK 1 in the money investment group on average increased the proposal by SEK 0.12.

${ }^{10}$ For convenience one proposal of SEK 49 is included in the SEK 50 category in Figure 2. A proposal of 30 is not shown in the figure, but is included in the analysis.

${ }^{11}$ One proposer in the monetary cost group made a proposal of SEK 63.5 and one proposer made a proposal of SEK 79. In Figure 3, these proposals are included in the SEK 65 and SEK 80 categories respectively. (All other proposals were divisible by 5.)
} 
an equal split (the relevant comparison when subjects round their offers to the nearest SEK 10). These numbers are in the same ballpark as those in Ellingsen and Johannesson (2005a).

We may also compare the distribution of proposals between the time investment group and the control group. The fraction of proposals of SEK 50 is almost identical in these two groups (about 30\% in each group). However, the fraction of high proposals is higher in the time investment group. The fraction of proposals at or above SEK 80 is $28 \%$ in the time investment group versus $7 \%$ in the control group $(p=0.029)$. It seems as if the proposers in the time investment group can roughly be divided into two categories. One category ignores the time cost when they make the bargaining proposal; the other category acts as if investment is monetary. The group that ignores the time cost is the same size as the group that propose an even split in the standard ultimatum game. It seems reasonable to guess that people who are fairminded in the ultimatum game are also generous with their time, but the only way to determine the overlap between the two groups is to have subjects participate in multiple treatments.

\subsection{Responder behavior}

The responders' average acceptance rate is about $90 \%$ in all three treatments (see Table 1). In Table 2 we report the results of a logistic regression testing whether the acceptance probability differs across treatments when we control for the proposal.

$* * * * * * * * * * * * * * *$ INSERT TABLE 2 ABOUT HERE*******************

For a given proposal, responders are somewhat more likely to accept the proposal if there has been a prior investment. The difference in acceptance probability is statistically significant at the ten percent level in our twosided test (and at the five percent level in a one-sided test, which is arguably more appropriate in this case), even if we do not pool the time and money treatment data. This finding is well in line with previous findings that responders consider the proposer's sunk costs to be relevant, even when these costs cannot be observed directly (Ellingsen and Johannesson, 2005a).

As expected, the acceptance probability decreases for higher proposals, and this effect is strongly significant $(p<0.001)$. The acceptance probability at a specific proposal is also significantly higher in the time investment group $(p=0.068)$ and the money investment group $(p=0.095)$ compared to the control group. The difference between the time investment group and the 
money investment group is small and not statistically significant $(p=0.710)$. We thus find no evidence that responder behavior differs between the money and time investment treatments. ${ }^{12}$

Looking at the raw data, there are no rejection of proposals below 70 in any of the treatments. It thus seems unlikely that all the subjects who propose 50 in the time treatment does so because they are afraid that more selfish proposals, such as 60 , would be rejected.

\section{Final remarks}

Our most notable finding is that a sizeable fraction of proposers require no compensation for their time investment, whereas almost all proposers require compensation for monetary investment. Our preferred explanation is that subjects are generally more prone to make non-monetary sacrifices than to make equivalent monetary sacrifices. Indeed, any alternative explanation would seem to involve some bias in our time cost elicitation procedure.

A natural avenue for further research is to identify the source of the money-time asymmetry. One possibility is that people have a greater tendency to behave generously in the time domain than in the money domain. Since proposals below 70 are never rejected in our data, it seems unlikely that a substantial fraction of the 50:50 splits are due to fears that greedier proposals will be rejected. A survey of donor attitudes by Lee, Piliavin, and Call (1999) supports the notion of domain-specific generosity. It finds that volunteering of time is more strongly affected by others' expectations than are gifts of blood and money. The authors speculate that the greater visibility of time donations make people behave more prosocially on this domain. ${ }^{13}$ If one would replicate the money-time asymmetry of our ultimatum bargaining experiment in an otherwise identical dictator game experiment, the case for the domain-specific generosity interpretation would be strengthened considerably.

Another explanation for our findings, suggested by an anonymous referee, is that subjects engage in mental accounting (Thaler, 1980, 1999) - account-

\footnotetext{
${ }^{12}$ As we only observe an accept/reject decision of each responder, the statistical power to detect differences in responder behavior is also limited.

${ }^{13}$ There is a significant economic literature on the non-monetary nature of gifts. See for example Camerer (1988), Waldfogel (1993), Prendergast and Stole (2001), Ellingsen and Johannesson (2004b).
} 
ing for monetary costs, but not time costs, when money is being divided. It seems feasible to conduct experiments that would discriminate between the two hypotheses, and we hope to see such experiments in the future.

\section{References}

Becker, G., DeGroot, M., Marschak, J., 1964. Measuring utility by a single-response sequential method. Behavioural Science 9, 226-232.

Becker, G.S., 1964. Human Capital. New York: Columbia University Press for the NBER.

Becker, G.S., 1965. A theory of the allocation of time. Economic Journal 75, 493-517.

Bohannan, P., 1963. Social Anthropology. New York: Holt, Rinehart and Winston.

Bohm, P., Lindén, J., Sonnegård, J., 1997. Eliciting reservation prices: Becker-DeGroot-Marschak mechanisms vs. markets. Economic Journal 107, 1079-1089.

Bolton, G., Ockenfels, A., 2000. ERC: A theory of equity, reciprocity, and competition. American Economic Review 90, 166-193.

Camerer, C.F., 1988: Gifts as economic signals and social symbols. American Journal of Sociology 94 (Supplement), S180-S214.

Camerer, C.F., 2003. Behavioral Game Theory: Experiments in Strategic Interaction. Princeton: Princeton University Press.

Charness, G. and Rabin, M., 2002. Understanding social preferences with simple tests. Quarterly Journal of Economics 117, 817-869.

D’Agostino, R.B., Chase, W., Belanger, A., 1988. The appropriateness of some common procedures for testing the equality of two independent binomial populations. American Statistician 42, 198-202.

Davidson, R., MacKinnon, J.G., 1999. The size distortion of bootstrap tests. Econometric Theory 15, 361-376. 
Efron, B., Tibshirani, R.J., 1993. An Introduction to the Bootstrap. Monographs on statistics and applied probability, No. 57. New York: Chapman and Hall.

Ellingsen, T., Johannesson, M., 2004a. Promises, threats, and fairness, Economic Journal 114, 397-420.

Ellingsen, T., Johannesson, M., 2004b. Generosity. Mimeo, Department of Economics, Stockholm School of Economics.

Ellingsen, T., Johannesson, M., 2005a. Sunk costs and fairness in incomplete information bargaining, Games and Economic Behavior 50, 155-177.

Ellingsen, T., Johannesson, M., 2005b. Does impartial deliberation breed fair behavior? An experimental test, Rationality and Society 17, 116136.

Fehr, E., Schmidt, K.M., 1999. A theory of fairness, competition, and cooperation. Quarterly Journal of Economics 114, 817-868.

Grether, D.M., Plott, C.R., 1979. Economic theory of choice and the preference reversal phenomenon. American Economic Review 69, 623638.

Lee, L.C., Piliavin, J.A., Call, V.R.A., 1999. Giving time, money, and blood: Similarities and differences. Social Psychology Quarterly 62, 276-290.

Prendergast, C., Stole, L., 2001. The non-monetary nature of gifts. European Economic Review 45, 1793-1810.

Rabin, Matthew., 1993. Incorporating fairness into game theory and economics. American Economic Review 83, 1281-1302.

Tetlock, P.E., Kristel, O.V., Elson, S.B., Green, M.C., Lerner, J.S., 2000. The psychology of the unthinkable: Taboo trade-offs, forbidden base rates, and heretical counterfactuals. Journal of Personality and Social Psychology 78, 853-870.

Thaler, R. H., 1980. Toward a positive theory of consumer choice. Journal of Economic Behavior and Organization 1, 39-60. 
Thaler, R. H., 1999. Mental accounting matters. Journal of Behavioral Decision Making 12, 183-206.

Waldfogel, J., 1993. The deadweight loss of Christmas. American Economic Review 83, 1328-1336.

Waltzer, M., 1983. Spheres of Justice. A Defence of Pluralism and Equality. Oxford: Blackwell.

Zelitzer, V.A.R., 1994. The Social Meaning of Money. New Jersey: Princeton University Press. 


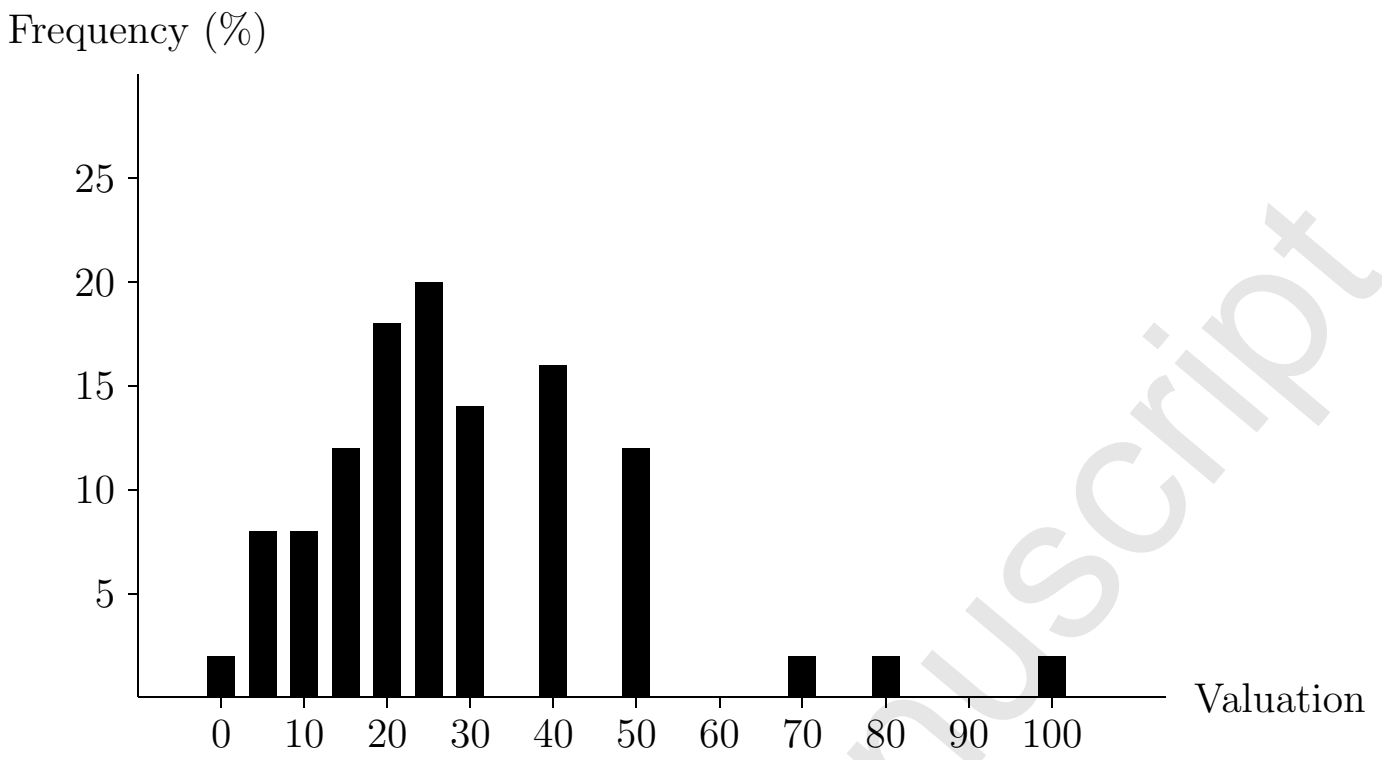

Figure 1: Valuations of time (SEK for 20 minutes) 


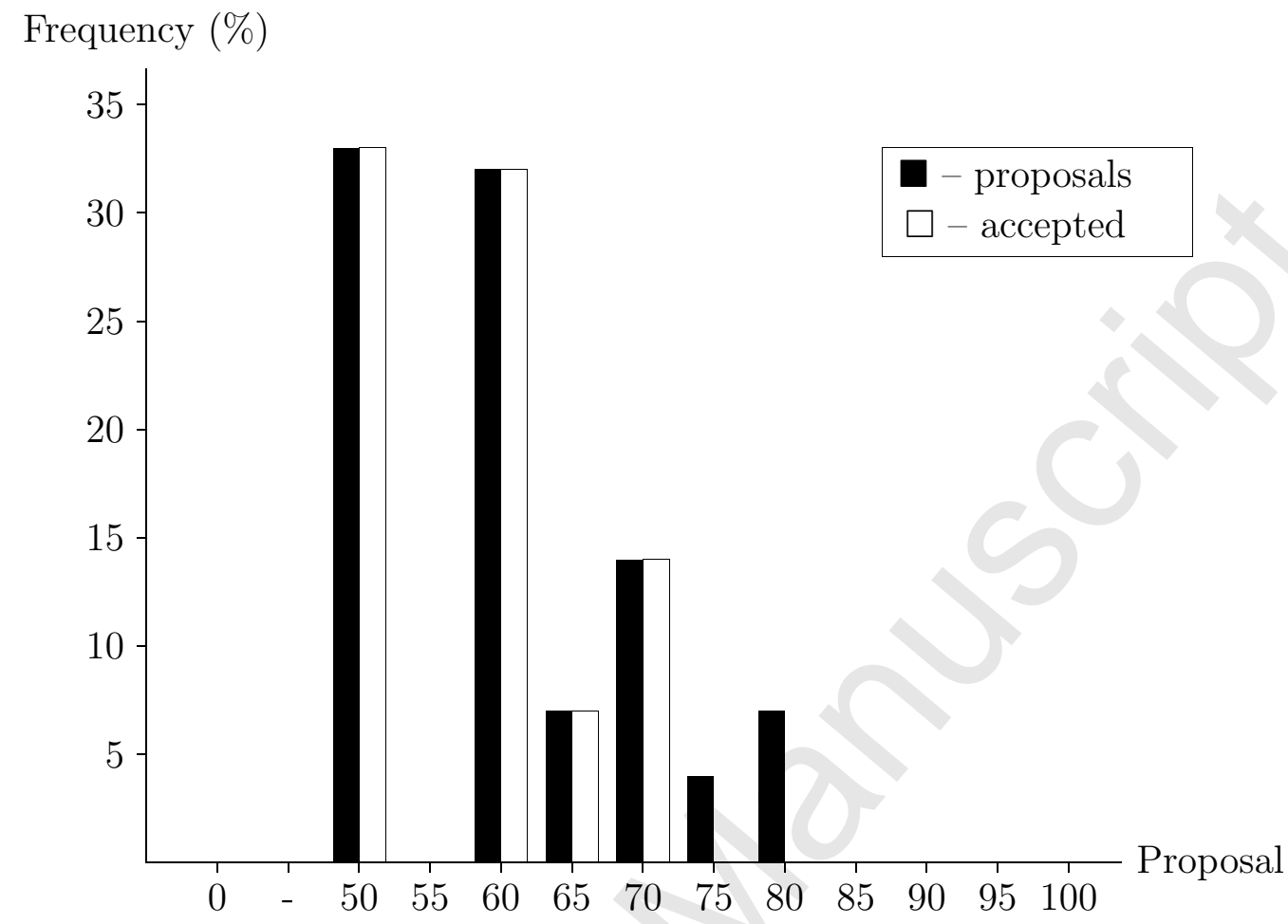

Figure 2: Proposals in the ultimatum game (control). 


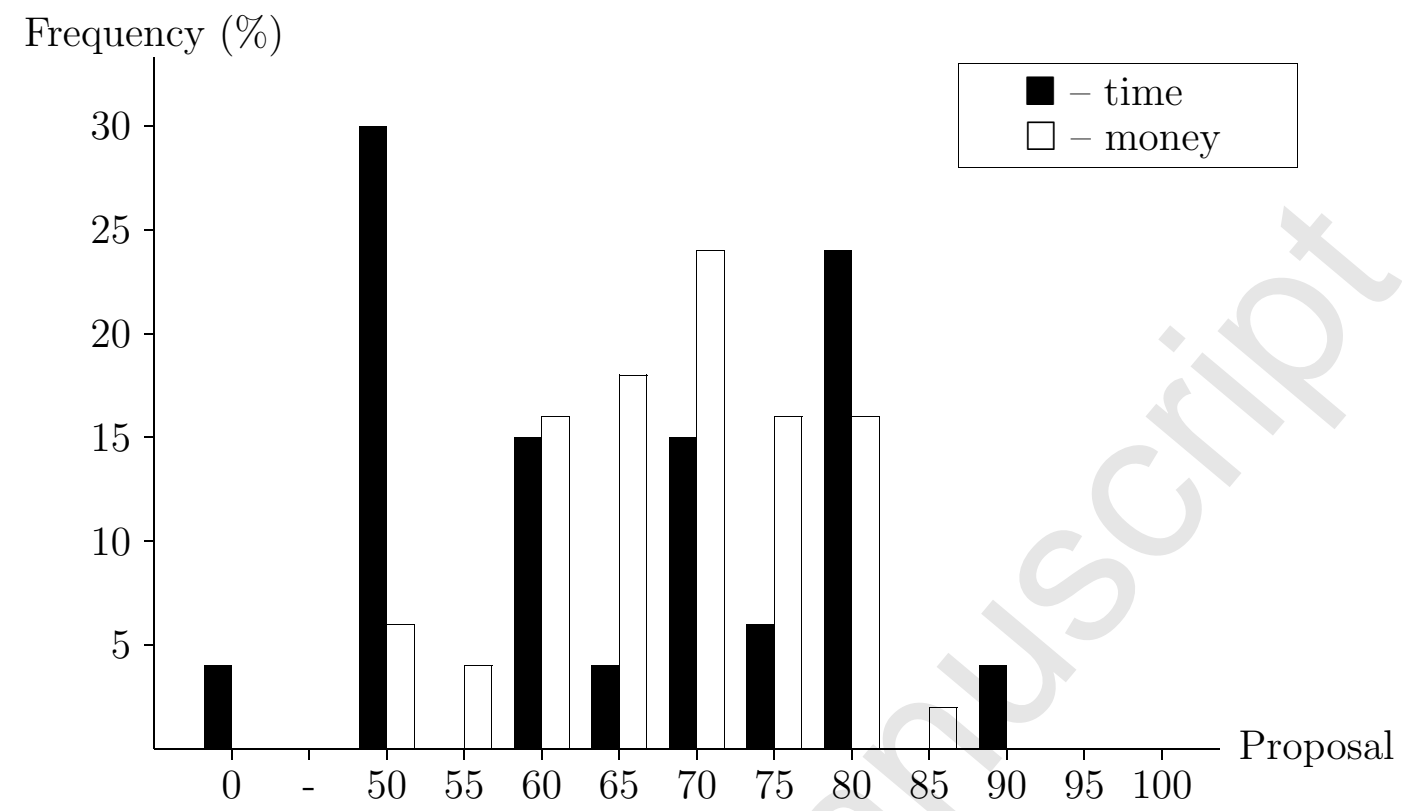

FigURE 3: Distribution of proposals in the two main treatments. 


\begin{tabular}{lrrrrrr}
\hline & \multicolumn{3}{c}{ Treatment } & \multicolumn{3}{c}{$p$-value of difference } \\
& Control & Time & Money & 1 vs 2 & 1 vs 3 & 2 vs 3 \\
\hline Number of pairs & 28 & 55 & 55 & & & \\
Proportion investors & 1.00 & 0.98 & 0.93 & 0.473 & 0.144 & 0.170 \\
Average proposal & 59.43 & 63.24 & 68.34 & 0.213 & 0.001 & 0.056 \\
Share of proposals $=50$ & 0.32 & 0.30 & 0.06 & 0.815 & 0.002 & 0.002 \\
Share of proposals $\geq 80$ & 0.07 & 0.28 & 0.18 & 0.029 & 0.197 & 0.217 \\
Acceptance rate & 0.89 & 0.89 & 0.90 & 0.957 & 0.898 & 0.827 \\
\hline
\end{tabular}

Table 1: Aggregate results 


\begin{tabular}{lrrr}
\hline Variable* $^{*}$ Coefficient & $p$-value & Marginal effect \\
\hline Constant & 19.695 & $<0.001$ & \\
Proposal & -0.260 & $<0.001$ & 0.0030 \\
Time investment dummy & 2.040 & 0.068 & 0.024 \\
Money investment dummy & 1.752 & 0.095 & 0.020 \\
\hline Number of pairs & 133 & \\
$\chi^{2}$ & 34.72 & \\
Log-likelihood & -27.39 & \\
McFadden pseudo $R^{2}$ & 0.39 & \\
Individual prediction (\%) & 91.73 \\
\hline \multicolumn{4}{c}{${ }^{2}$ Baseline category is costless investment (control). }
\end{tabular}

Table 2: Logistic regression of acceptance probability. 


\section{APPENDIX 1: EXPERIMENTAL INSTRUCTIONS}

The original instructions were in Swedish. This appendix reprints a translation of the instructions used in the three experimental treatments (the control group, the time investment group, and the money investment group). The instructions for the estimation of the time cost with the BDM mechanism are also reprinted below.

\section{INSTRUCTIONS (The control group)}

Thank you for agreeing to participate in this experiment. As a compensation for participating you (and the other participants in the experiment) will receive SEK 100. This compensation of SEK 100 plus the profit you make during the experiment will be paid towards the end of the experiment. In the experiment each of you will be paired with a trading partner who is in another room. Neither of you will be told who the trading partner is either during or after the experiment. There is the same number of persons in each room (A and B). This is room A (B). Every person in room A and B has been given these instructions and a number that shows which pair he/she belong to. The experiment consists of two stages.

At stage 1 every person in room A has to decide whether to carry out a costless investment or not. If the person in room A decides to make the investment revenue of SEK 100 is created for the pair that the person belongs to, and the person in room A makes a proposal for how to divide the revenue of SEK 100 between himself and the trading partner in room B.

In stage 2 the trading partner in room B can accept or reject this proposal. The negotiation has two possible outcomes:

(i) The person in room B accepts the proposed division. The SEK 100 are then divided according to the proposal.

(ii) The person in room B rejects the proposed division. Each person then receives nothing.

The experiment is carried out in the following way. At stage 1 every person in room A decides whether to carry out the investment or not. This is done by choosing "yes" or "no" on the form marked "Investment decision" (that has been given to all persons in room A). Persons in room A that have chosen to make the investment also write down their proposed divisions of the revenue on the same form. In stage 2 the forms are collected and handed out to each trading partner in room B. For the pairs where investment was chosen the trading partner accepts or rejects the proposed division by marking the respective "Accept" or "Reject” box. The forms are then collected again and handed out to the persons in room A.

In the protocol on page 2 of these instructions both persons record the outcome of the experiment; i.e. the investment decision, the proposed division, if the proposed division was accepted or not, and their profit from the experiment (that is given by the proposed division if the proposal was accepted; if the proposal was rejected the profit is SEK 0). For persons in pairs where no investment was made, the profit from the experiment is SEK 0. Every person then receives his or her profit from the experiment plus the SEK 100 participation fee. 


\section{PROTOCOL}

The person in room A chose to make the investment:

yes......

no.....

If the person in room A chose not to make the investment, your profit of the experiment is SEK 0 and the negotiation is cancelled. The remainder of the protocol should only be filled in if you belong to a pair where the person in room A chose to make the investment.

Proposed division from the person in room $\mathbf{A}$ of the revenue of SEK 100:

The person in room A receives..............SEK

The person in room $\mathrm{B}$ receives..............SEK

The proposal was accepted by the person in room B: yes..... no.......

Your profit from the experiment

SEK*

(If the proposed division was rejected your profit from the experiment is SEK 0. If the proposed division was accepted your profit from the experiment is equal to the proposed division.)

* Note that each person that participates in the experiment in both room A and room B apart from the profit of the experiment will also receive a compensation of SEK 100 for participating in the experiment. 
INSTRUCTIONS (The time investment group)

Thank you for agreeing to participate in this experiment. As a compensation for participating you (and the other participants in the experiment) will receive SEK 100. This compensation of SEK 100 plus the profit you make during the experiment will be paid towards the end of the experiment. In the experiment each of you will be paired with a trading partner who is in another room. Neither of you will be told who the trading partner is either during or after the experiment. There is the same number of persons in each room (A and B). This is room A (B). Every person in room A and B has been given these instructions and a number that shows which pair he/she belong to. The experiment consists of two stages.

At stage 1 every person in room A has to decide whether to carry out an investment or not (the investment cost, that is paid by the person in room $\mathrm{A}$, is described below). If the person in room A decides to make the investment revenue of SEK 100 is created for the pair that the person belongs to, and the person in room $A$ makes a proposal for how to divide the revenue of SEK 100 between himself and the trading partner in room B.

In stage 2 the trading partner in room B can accept or reject this proposal. The negotiation has two possible outcomes:

(i) The person in room B accepts the proposed division. The SEK 100 are then divided according to the proposal.

(ii) The person in room B rejects the proposed division. Each person then receives nothing.

The investment cost is that the person in room A stays after the end of the experiment for 20 minutes. To fill out the time during these 20 minutes each person in room A that carry out the investment will be given a questionnaire to complete. (Observe that it is only the person in room A that can make the investment and who pays the entire investment cost. The trading partner in room B always leaves directly after the end of the experiment.)

The experiment is carried out in the following way. At stage 1 every person in room A decides whether to carry out the investment or not, i.e. if he/she will stay for 20 minutes after the end of the experiment. This is done by choosing "yes" or "no" on the form marked "Investment decision" (that has been given to all persons in room A). Persons in room A that have chosen to make the investment also write down their proposed divisions of the revenue on the same form. In stage 2 the forms are collected and handed out to each trading partner in room B. For the pairs where investment was chosen the trading partner accepts or rejects the proposed division by marking the respective "Accept" or "Reject" box. The forms are then collected again and handed out to the persons in room A.

In the protocol on page 2 of these instructions both persons record the outcome of the experiment; i.e. the investment decision, the proposed division, if the proposed division was accepted or not, and their profit from the experiment (that is given by the proposed division if the proposal was accepted; if the proposal was rejected the profit is SEK 0). For persons in pairs where no investment was made, the profit from the experiment is SEK 0 . Every person then receives his or her profit from the experiment plus the SEK 100 participation fee. Thereafter all participants can leave, except the persons in room A that chose to make the investment. These persons stay for 20 minutes (irrespectively of if their proposed division was accepted or not). 


\section{PROTOCOL}

The person in room A chose to make the investment:

yes.....

no.....

If the person in room A chose not to make the investment, your profit of the experiment is SEK 0 and the negotiation is cancelled. The remainder of the protocol should only be filled in if you belong to a pair where the person in room A chose to make the investment.

Proposed division from the person in room $\mathbf{A}$ of the revenue of SEK 100:

The person in room A receives..............SEK

The person in room $\mathrm{B}$ receives..............SEK

The proposal was accepted by the person in room B: yes..... no.......

Your profit from the experiment

SEK*

(If the proposed division was rejected your profit from the experiment is SEK 0. If the proposed division was accepted your profit from the experiment is equal to the proposed division.)

* Note that each person that participates in the experiment in both room A and room B apart from the profit of the experiment will also receive a compensation of SEK 100 for participating in the experiment. 


\section{INSTRUCTIONS (The money investment group)}

Thank you for agreeing to participate in this experiment. As a compensation for participating you (and the other participants in the experiment) will receive SEK 100. This compensation of SEK 100 plus/minus the profit/loss you make during the experiment will be paid towards the end of the experiment. In the experiment each of you will be paired with a trading partner who is in another room. Neither of you will be told who the trading partner is either during or after the experiment. There is the same number of persons in each room (A and B). This is room A (B). Every person in room A and B has been given these instructions and a number that shows which pair he/she belong to. The experiment consists of two stages.

At stage 1 every person in room A has to decide whether to carry out an investment or not (the investment cost, that is paid by the person in room $\mathrm{A}$, is described below). If the person in room $\mathrm{A}$ decides to make the investment revenue of SEK 100 is created for the pair that the person belongs to, and the person in room $A$ makes a proposal for how to divide the revenue of SEK 100 between himself and the trading partner in room B.

In stage 2 the trading partner in room B can accept or reject this proposal. The negotiation has two possible outcomes:

(i) The person in room B accepts the proposed division. The SEK 100 are then divided according to the proposal.

(ii) The person in room B rejects the proposed division. Each person then receives nothing.

The investment cost varies between different persons in room A and is given on the form marked "Investment cost" (that has been given to all persons in room A). (Observe that it is only the person in room A that can make the investment and who pays the entire investment cost.) The exact size of the investment cost is not revealed to the person in room $B$. The investment cost for each person in room A has been determined in the following manner. In a separate group in the experiment 50 individuals have valued their cost of staying after the end of the experiment for 20 minutes (and they were informed that they would complete a questionnaire during these 20 minutes to pass the time). The valuation was done in a way so that every person had an incentive to reveal his/her true cost. For every person in room A we have randomly chosen the cost of giving up 20 minutes for one of these 50 individuals and used this cost as the investment cost.

The experiment is carried out in the following way. At stage 1 every person in room A decides whether to carry out the investment or not. This is done by choosing "yes" or "no" on the form marked "Investment decision" (that has been given to all persons in room A). Persons in room A that have chosen to make the investment also write down their proposed divisions of the revenue on the same form. In stage 2 the forms are collected and handed out to each trading partner in room B. For the pairs where investment was chosen the trading partner accepts or rejects the proposed division by marking the respective "Accept" or "Reject" box. The forms are then collected again and handed out to the persons in room A.

In the protocol on page 2 of these instructions both persons record the outcome of the experiment; i.e. the investment decision, the proposed division, if the proposed division was accepted or not, and their revenue from the negotiation (that is given by the proposed division if the proposal was accepted; if the proposal was rejected the revenue is SEK 0). Finally each person calculates their profit from the experiment and writes it down on the protocol. For persons in room A the profit is equal to the revenue of the negotiation minus the investment cost. For persons in room B the profit of the experiment is equal to the revenue of the negotiation. For persons in pairs where no investment was made, the profit from the experiment is SEK 0. Every person then receives his or her profit from the experiment plus the SEK 100 participation fee. Thereafter all participants can leave. 


\section{PROTOCOL}

The person in room A chose to make the investment:

yes.....

no.....

If the person in room A chose not to make the investment, your profit of the experiment is SEK 0 and the negotiation is cancelled. The remainder of the protocol should only be filled in if you belong to a pair where the person in room A chose to make the investment.

Proposed division from the person in room $\mathbf{A}$ of the revenue of SEK 100:

The person in room A receives...............SEK

The person in room $\mathrm{B}$ receives..............SEK

The proposal was accepted by the person in room B: yes..... no......

Your revenue from the negotiation.

SEK

(If the proposed division was rejected your revenue from the negotiation is SEK 0. If the proposed division was accepted your revenue from the negotiation is equal to the proposed division.)

Your profit from the experiment.

SEK*

(If you are in room A your profit from the experiment is equal to your revenue from the negotiation minus the investment cost and if you are in room B your profit is equal to your revenue from the negotiation.)

* Note that each person that participates in the experiment in both room A and room B apart from the profit of the experiment will also receive a compensation of SEK 100 for participating in the experiment. 
INSTRUCTIONS (Estimation of time cost with the BDM mechanism)

In this part of the experiment we are interested in your (and the other participants) valuation of the cost of staying after the end of the experiment for 20 minutes. We will offer you a specific compensation to stay after the end of the experiment for 20 minutes (to pass the time during these 20 minutes you will be given a questionnaire to complete).

On the enclosed form marked "Time cost” you will fill out the smallest compensation you accept to stay after the end of the experiment for 20 minutes. The forms are then collected.

Thereafter we will draw the price that we are willing to pay for you to stay after the end of the experiment for 20 minutes. The price is drawn randomly from a box that contain notes with prices from SEK 0 and upwards in SEK 5 increments: i.e. SEK 0, SEK 5, SEK 10, SEK 15, SEK 20, etc. upwards to the highest possible price. The highest possible price is equal to what we think is the highest possible reasonable valuation of the cost of giving up 20 minutes. In the random draw of the price one of you will draw a note with a price. After this draw you are welcome to inspect all the notes in the box.

If the randomly drawn price (SEK X) is higher than or equal to the time cost you stated on the form we will pay you the drawn price (SEK X) and you will have to stay for 20 minutes. If the randomly drawn price (SEK X) is lower than the time cost you stated on the form, you can leave directly after the end of the experiment.

Note that it is in your own best interest to state your time cost as accurately as possible, i.e. to be honest. This is because you cannot affect how much you get paid for staying for 20 minutes as this price is randomly drawn. The only thing you can affect is if you will stay for 20 minutes or not at different prices. 


\section{TIME COST}

What is the smallest compensation you accept to stay after the end of the experiment for 20 minutes? .SEK 


\section{APPENDIX 2: DATA TABLES}

Estimation of time cost (20 minutes) with the Becker-DeGroot-Marschak (BDM) mechanism. $\mathrm{n}=56$.

Number (\%)

0

$1(2)$

5

$4(7)$

10

4(7)

15

6(11)

20

9(16)

25

10(18)

30

7(12)

39

1(2)

40

5(9)

49

1(2)

50

5(9)

70

1(2)

80

1(2)

100

1(2) 
Bargaining behavior in the ultimatum game. Zero investment cost. $\mathrm{n}=28$.

Proposed division $\quad$ Number of pairs(\%) Number of accepted proposals

Investor Trading partner

\begin{tabular}{llll}
\hline 30 & 70 & $1(4)$ & 1 \\
49 & 51 & $1(4)$ & 1 \\
50 & 50 & $8(29)$ & 8 \\
60 & 40 & $9(32)$ & 9 \\
65 & 35 & $2(7)$ & 2 \\
70 & 30 & $4(14)$ & 4 \\
75 & 25 & $1(4)$ & 0 \\
80 & 20 & $2(7)$ & 0 \\
\hline
\end{tabular}


Bargaining behavior in the ultimatum game. Time investment cost. $\mathrm{n}=54$.

Proposed division $\quad$ Number of pairs(\%) Number of accepted proposals Investor Trading partner

\begin{tabular}{llll}
\hline 0 & 100 & $2(4)$ & 2 \\
50 & 50 & $16(30)$ & 16 \\
60 & 40 & $8(15)$ & 8 \\
65 & 35 & $2(4)$ & 2 \\
70 & 30 & $8(15)$ & 7 \\
75 & 25 & $3(6)$ & 3 \\
80 & 20 & $13(24)$ & 10 \\
90 & 10 & $2(4)$ & 0 \\
\hline
\end{tabular}


Bargaining behavior in the ultimatum game. Money investment cost. $\mathrm{n}=51$.

Proposed division $\quad$ Number of pairs(\%) Number of accepted proposals Investor Trading partner

\begin{tabular}{llll}
\hline 50 & 50 & $3(6)$ & 3 \\
55 & 45 & $2(4)$ & 2 \\
60 & 40 & $8(16)$ & 8 \\
63.5 & 37.5 & $1(2)$ & 1 \\
65 & 35 & $8(16)$ & 8 \\
69 & 31 & $2(4)$ & 2 \\
70 & 30 & $10(20)$ & 9 \\
75 & 25 & $8(16)$ & 7 \\
79 & 21 & $1(2)$ & 0 \\
80 & 20 & $7(14)$ & 5 \\
85 & 15 & $1(2)$ & 1 \\
\hline
\end{tabular}

